\title{
Publisher Correction: Probing dynamics in quantum materials with femtosecond X-rays
}

Michele Buzzi, Michael Först, Roman Mankowsky and Andrea Cavalleri

Nature Reviews Materials (2018) https://doi.org/10.1038/s41578-018-0024-9

Published online 01 June 2018

This article was originally published with an error in the main text. The original sentence, "The on-resonance intensity includes a charge order contribution (grey shaded region, FIG. 2b), which disappears on a timescale shorter than that of the off-resonance intensity and is sensitive only to structural dynamics", should have read: "The on-resonance intensity includes a charge order contribution (grey shaded region, FIG. 2b) that disappears on a timescale shorter than that of the off-resonance intensity, which is sensitive only to structural dynamics."

We apologize to the readers for this error.

https://doi.org/10.1038/s41578-018-0028-5 I Published online 12 June 2018 